\title{
INTRODUCING FULLY UP-SEMIGROUPS ${ }^{1}$
}

\author{
AIYARED IAMPAN ${ }^{2}$ \\ Department of Mathematics, School of Science \\ University of Phayao, Phayao 56000, Thailand \\ e-mail: aiyared.ia@up.ac.th
}

\begin{abstract}
In this paper, we introduce some new classes of algebras related to UPalgebras and semigroups, called a left UP-semigroup, a right UP-semigroup, a fully UP-semigroup, a left-left UP-semigroup, a right-left UP-semigroup, a left-right UP-semigroup, a right-right UP-semigroup, a fully-left UP-semigroup, a fully-right UP-semigroup, a left-fully UP-semigroup, a right-fully UP-semigroup, a fully-fully UP-semigroup, and find their examples.
\end{abstract}

Keywords: semigroup, UP-algebra, fully UP-semigroup.

2010 Mathematics Subject Classification: 08A99, 03G25.

\section{REFERENCES}

[1] S.S. Ahn and Y.H. Kim, On BE-semigroups, Int. J. Math. Math. Sci. (2011) Article ID $676020,2011$. doi:10.1155/2011/676020

[2] J.C. Endam and J.P. Vilela, On JB-semigroups, Appl. Math. Sci. 9 (2015) 29012911. doi:10.12988/ams.2015.46427

[3] A. Iampan, A new branch of the logical algebra: UP-algebras, J. Algebra Relat. Top. 5 (2017) 35-54. doi:10.22124/JART.2017.2403

[4] Y.B. Jun, S.M. Hong and E.H. Roh, BCI-semigroups, Honam Math. J. 15 (1993) 59-64.

[5] Y.B. Jun, E.H. Roh and X.L. Xin, I-ideals generated by a set in IS-algebras, Bull. Korean Math. Soc. 35 (1998) 615-624.

\footnotetext{
${ }^{1}$ This work was financially supported by the University of Phayao.

${ }^{2}$ Corresponding author.
} 
[6] Y.B. Jun, X.L. Xin and E.H. Roh, A class of algebras related to BCI-algebras and semigroups, Soochow J. Math. 24 (1998) 309-321.

[7] F.F. Kareem and E.R. Hasan, On KU-semigroups, Int. J. Sci. Nat. 9 (2018) 79-84.

[8] K.H. Kim, On structure of KS-semigroups, Int. Math. Forum 1 (2006) 67-76.

[9] S.M. Lee and K.H. Kim, A note on HS-algebras, Int. Math. Forum 6 (2011) 15291534.

[10] J.K. Park, W.H. Shim and E.H. Roh, On isomorphism theorems in IS-algebras, Soochow J. Math. 27 (2001) 153-160.

[11] F. Sultana and M.A. Chaudhary, BCH-semigroup ideals in BCH-semigroups, Palestine J. Math. 5 (2016) 1-5.

Received 9 September 2018

Revised 21 September 2018

Accepted 26 September 2018 